\title{
HALOS, STARBURSTS, AND SUPERBUBBLES IN SPIRALS
}

\author{
Joel N. Bregman \\ Department of Astronomy, University of Michigan, Ann Arbor, MI 48109-1090 \\ jbregman@astro.lsa.umich.edu
}

\begin{abstract}
Detectable quantities of interstellar material are present in the halo of the Milky Way galaxy and in a few edge-on spiral galaxies, largely in the form of neutral atomic gas, warm ionized material, and cosmic rays. Theoretical and observational arguments suggest that million degree gas should be present also, so sensitive ROSAT observations have been made of the large nearby edgeon spiral galaxies for the purpose of detecting hot extraplanar gas. Of the six brightest non-starburst edge-on galaxies, three exhibit extraplanar X-ray emission: NGC 891, NGC 4631, and NGC 4565. In NGC 891, the extended emission has a density scale height of $7 \mathrm{kpc}$ and an extent along the disk of $13 \mathrm{kpc}$ in diameter. This component is close to hydrostatic equilibrium, has a luminosity of $4.4 \times 10^{39} \mathrm{erg} \mathrm{s}^{-1}$, and a mass of $10^{8} \mathrm{M}_{\odot}$. Extended and structured extraplanar hot gas is seen around the interacting edge-on spiral NGC 4631, with $\mathrm{X}$-ray emission associated with a giant loop of $\mathrm{H} \alpha$ and $\mathrm{HI}$ emission; spurs of Xray emission extending from the disk are seen also. Hot gas is expected to enter the halo through superbubble breakout, and a search for superbubbles in normal spiral galaxies have shown that these phenomena are present, but of low surface brightness and are detected in only a few instances.

Unlike the normal spiral galaxies where the gas is bound to the systems, the hot gas in starburst galaxies is being expelled. In M82, the X-ray emission lies in loosely-defined cones that extend to a projected radius of $6.5 \mathrm{kpc}$. The decrease of the surface brightness with position from the nucleus is consistent with adiabatic expansion of gas flowing from the central region of the galaxy.
\end{abstract}

\section{The Theoretical Expectations For Hot Gas In And Around Spiral Galaxies}

An abundance of hot gas is the expected manifestation of an active star formation region. During their brief life, high mass stars $\left(>5 \mathrm{M}_{\odot}\right)$ drive strong stellar winds at speeds in the thousand $\mathrm{km} \mathrm{s}^{-1}$ range, which produces a hot bubble of gas in the vicinity of the star. At the end of their lives, these high mass stars become supernovae, depositing into the interstellar medium 3-4 times more energy than during the wind phase, along with a considerable amount of processed materials. Usually, high-mass stars occur in associations where there might be 30 supernova progenitors, and the mean time between supernova explosions in the association is less than the cooling time of the hot material. This leads to the collective growth of a hot bubble (or "superbubble"), which can become large enough to break out of the disk (review of these phenomena by Tenorio-Tagle and Bodenheimer 1988).

Breakout of hot bubbles depends upon the number of supernova per unit time (the input "luminosity", $\mathrm{L}_{*}$ ) and the mean density of the gas, but there are three possible scenarios. If the input energy rate $L_{*}$ is too low or (and) too short in duration, the bubble radiates its thermal energy before becoming large enough to break out of the disk. In this event, one might see bubbles of $\mathrm{X}$-ray emitting material that were roughly spherical and confined to the disk of 
a spiral galaxy. When the input energy rate $L_{*}$ and the total energy released over time is sufficient for the bubble size to exceed the thickness of the cold disk of gas, the bubble grows in an irregular fashion, becoming elongated in the direction perpendicular to the disk. When the growth of the bubble in the vertical direction begins to accelerate, the cold confining shell becomes unstable to Rayleigh-Taylor instabilities, and the hot gas escapes the bubble, expanding into the halo or extraplanar region of the galaxy (Tomisaka and Ikeuchi 1986; MacLow, McCray, and Norman 1989).

The fate of hot gas that has escaped the disk depends upon its thermal energy at the time of breakout and the depth of the potential well of the galaxy (Chevalier and Oegerle 1979; Bregman 1980). If the sum of the thermal, kinetic, and gravitational energies is negative, the gas remains bound to the galaxy and will try to establish hydrostatic equilibrium with the galactic gravitational field. The timescale for this to occur is a sound-crossing time over a length scale of the pressure scale height that the gas would have in hydrostatic equilibrium. Gas that cools slowly (cooling time longer than a sound-crossing time) will have a thickness, perpendicular to the disk that is given by hydrostatic theory, for which the length scale depends only on the temperature. This is a simple prediction that can be tested by ROSAT observations, as will be discussed. However, if the cooling time is shorter than a sound-crossing time, the thickness of the Xray emitting material will be smaller than the hydrostatic value, and the $X$-ray emission will probably appear to be irregular or structured in appearance (either of these two possibilities is often referred to as galactic fountains).

Gas with positive total energy will escape freely, being unbound to the system. Some thermal energy is converted to kinetic energy and the temperature of the gas drops rapidly through adiabatic expansion. Both of these mechanisms lower the amount of radiated $\mathrm{X}$-ray emission, compared to gravitationally bound gas, so systems with unbound outflows (galactic winds) would be more difficult to detect unless $L_{*}$ was very high. Several of these theoretical expectations are clear and easy to test, and this has been a goal of X-ray astronomy.

\section{The Observational Picture Prior To ROSAT}

There were abundant $X$-ray observations of individual supernova remnants prior to ROSAT, but the observations of individual bubbles were less plentiful. Only a few superbubbles were known, and all but one in our Galaxy. In our galaxy, the Sun seems to lie in a hot low density region of size 100 pc (Fried et al. 1980; Cox and Reynolds 1987 and references therein). It is generally easier to study superbubbles that we do not live in, such as the Cygnus superbubble (Cash et al. 1980), which has a diameter of $450 \mathrm{pc}$, a luminosity of $5 \times 10^{36} \mathrm{erg} \mathrm{s}^{-1}$, and a temperature of $2 \times 10^{6} \mathrm{~K}$. Singh et al. (1987) found a large superbubble in the LMC $(1.0 \times 1.4 \mathrm{kpc})$ associated with a shell of optical nebulosity (Shapley III) with an X-ray luminosity of $3.4 \times 10^{37} \mathrm{erg} \mathrm{s}^{-1}$; this lies in an HI hole (also, see Wang and Helfand 1991a,b).

On theoretical grounds, it is expected that a neutral shell of gas surrounds a superbubble. Giant HI shells and partial shells with radii of 100-1000 pc are found with relative ease (compared with $\mathrm{X}$-ray discoveries) and have been seen in our galaxy by Heiles $(1979,1984)$ and Colomb, Poppel, and Heiles (1980). In M31 (Brinks and Bajaja 1986) and in M33 (Deul 1988; Deul and den Hartog 1989), "holes" in the HI disk are found with sizes exceeding 100 pc. It has been suggested that these shells are the HI signatures of superbubbles (e.g., McCray 
and Kafatos 1987), although for a convincing case to be made, one should also find a bubble of hot X-ray emitting gas within the HI shells.

Regarding the breakout of gas around normal galaxies, previous X-ray observations have been disappointing. None of the normal edge-on galaxies observed with the Einstein Observatory displayed detectable emission (Bregman and Glassgold 1982; Fabbiano 1989). Furthermore, spiral disks, when viewed face-on, showed little evidence of diffuse emission that might be associated with an unresolved ensemble of hot gas bubbles.

The only spiral galaxies that displayed extended X-ray emission were starburst galaxies. In these systems, the energy input rate $L_{*}$ is estimated to be very high (0.1 SN $\left.\mathrm{yr}^{-1}\right)$ so that hot gas is produced in abundance and with enough energy to flow outward as a wind. The best examples of these starburst galaxies are M82 and NGC 253, and we will concentrate on the former as an example. The X-ray map made with the HRI on the Einstein Observatory (Watson, Stanger, and Griffiths 1984) shows several point sources distributed through the star-forming region (about $30^{\prime \prime}$ in size) as well as a diffuse X-ray halo. This $\mathrm{X}$-ray halo is extended along the minor axis $\left(3^{\prime}\right.$ toward the SE, 1.7-2.5' toward the NE) much more so than along the major axis (half-width $<1.4^{\prime}$ ). Deeper, low resolution imaging with the Einstein IPC suggests that the X-ray halo may extend as far as $9^{\prime}$ from the nucleus (Fabbiano 1989).

The recent observations with the ROSAT HRI and PSPC have revised our perspective of these phenomena, especially in the areas of edge-on spiral galaxies and starburst galaxies.

\section{The New Perspective Provided By ROSAT}

\subsection{Superbubbles}

Observations of superbubbles in our Galaxy have been assisted greatly by the ROSAT All-Sky Survey, which have been discussed in detail in this meeting by Snowden (1994) and Plucinsky et al. (1994). For normal external spiral galaxies, several of the presentations at this meeting indicate that diffuse emission is present, often in spiral arm regions where star formation activity is high (M33, Long et al. 1994; NGC 6946, Schlegel 1994; M101, Murphy and Chu 1994; the LMC, Wang 1994). Individual supernovae are seen occasionally, especially in the nearby systems, and several star forming associations are detected, sometimes extended on the size scale expected from superbubbles (M33, Schulman and Bregman 1994; M101, Murphy and Chu 1994). Most of these superbubbles are weak detections, so the ROSAT observations will serve to determine the high end of their luminosity function.

\subsection{Extraplanar Gas Around Spiral Galaxies With Normal Star Formation}

The most direct and unambiguous approach for detecting halo or extraplanar gas is to observe edge-on galaxies without warps, or at least without warps that are projected onto the inner part of the galaxy, where extraplanar gas is expected to be most common. Also, one wishes to observe "normal" galaxies, as opposed to interacting systems, a qualification that will simplify the interpretation, when emission is detected. Of the nine edge-on galaxies that have been detected, three are starburst systems (NGC 3079, NGC 2146, and NGC 
3628 ), another is interacting with neighboring systems (NGC 4631), and the remaining five are nearly normal galaxies (NGC 891, NGC 4244, NGC 5907, NGC 4565 , and NGC 5529). These galaxies are the nearest edge-on galaxies and are typically $5^{\prime}-15^{\prime}$ in optical diameter.

Many of these edge-on galaxies were observed, beginning 15 years ago, in the radio region in an effort to search for radio halos and evidence that magnetic fields and relativistic particles could rise above the thin disk of cold interstellar gas. Some, such as NGC 891 and NGC 4631 have prominent radio halos extending a few kiloparsecs above the disk (Sancisi and Allen 1979; Rupen 1991). Many of these same galaxies were observed in the optical region in the light of $\mathrm{H} \alpha$, and considerable diffuse emission was detected in NGC 891 and NGC 4631, but not in NGC 4244, NGC 5907, or NGC 4565 (Rand, Kulkarni, and Hester 1990).

Many, if not most galaxies fail to show extraplanar X-ray emission. PSPC observations indicate null detections of X-rays from NGC 4244 and NGC 5529, while emission from the plane is detected from NGC 5907 (Frenk, private communication; Pietsch 1993). However, three systems show evidence for extraplanar gas - NGC 891, NGC 4631, and NGC 4565. The case of NGC 4631 is discussed separately in these proceedings by Walterbos (1994), but to summarize the morphological appearance, the extraplanar gas is semi-spherical, but with localized bright regions, possibly caused by filaments. The luminosity is 6 $\times 10^{39} \mathrm{erg} \mathrm{s}^{-1}$, and the temperature of the gas is $3.5 \times 10^{6} \mathrm{~K}$, although there may be a cooler gas component, which is difficult to quantify. A second galaxy with diffuse emission is NGC 4565 (Pietsch 1993), but these data have been obtained only recently and a detailed analysis is not yet complete.

The third galaxy with detected extraplanar X-ray emission is NGC 891, a spiral galaxy that is nearly an exact twin of our own Milky Way galaxy, with a rotational velocity of $225 \mathrm{~km} \mathrm{~s}^{-1}$, an inclination of $88.6-90^{\circ}$, and a distance of $10 \mathrm{Mpc}$. NGC 891 has a more extensive $\mathrm{H} \alpha$ halo than any of the other normal or near-normal galaxies, and there is a close coincidence between the $\mathrm{H} \alpha$ halo and the radio halo. Our $25 \mathrm{ksec}$ PSPC observation of NGC 891 revealed a few point sources in the disk (the brightest being SN 1986J; Bregman and Pildis 1992) plus extended diffuse emission with a FWHM of $100^{\prime \prime}(4.8 \mathrm{kpc})$ and a maximum detectable halo size of twice that value (Bregman and Pildis 1994). The diffuse emission extends $130-140^{\prime \prime}(6.5 \mathrm{kpc})$ along the disk relative to the center of the galaxy (Fig. 1), and there is an additional "plume" of emission extending about $130^{\prime \prime}(6.1 \mathrm{kpc})$ above the disk and from the nuclear region (this plume has no equivalent $\mathrm{H} \alpha$ or radio feature). This X-ray emission has a similar, if not somewhat larger extent when compared to the $\mathrm{H} \alpha$ and radio halo, suggesting that the various types of interstellar medium are cospatial in a general sense.

Spectral fits to the data require a two temperature fit, where the lower temperature is approximately $0.3 \mathrm{keV}\left(3.6 \times 10^{6} \mathrm{~K}\right.$; associated luminosity is $\left.4.4 \times 10^{39} \mathrm{erg} \mathrm{s}^{-1}\right)$ while the higher temperature is $>2 \mathrm{keV}\left(>2.4 \times 10^{7} \mathrm{~K}\right.$; associated luminosity of $5.2 \times 10^{39} \mathrm{erg} \mathrm{s}^{-1}$ ). We interpret this lower temperature component as originating from the hot gas while the higher temperature component originates from an ensemble of X-ray binary stars in the disk. For a filling factor of unity, the hot gas has a density scale height of $7 \mathrm{kpc}$ and a density near the disk of $2 \times 10^{-3} \mathrm{~cm}^{-3}$, which leads to a total hot gas mass of 1 $\times 10^{8} \mathrm{M}_{\odot}$. This gas is most likely bound to NGC 891 in that the density scale height is the value expected for gas in hydrostatic equilibrium. 

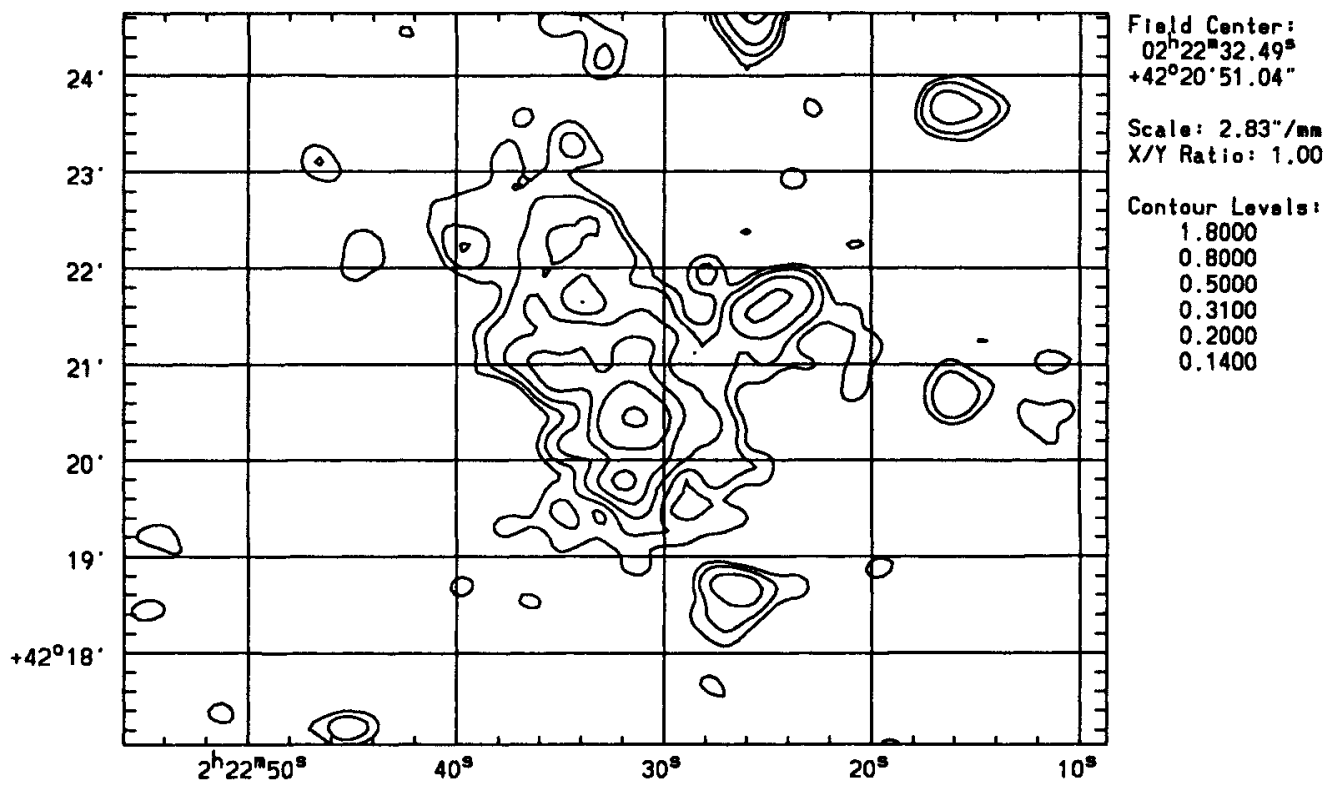

Figure 1. The PSPC contour map of NGC 891 after removal of the point source SN 1986J. The midplane of this edge-on galaxy is aligned N/NE - S/SW and the X-ray emission has a FWHM perpendicular to the disk of 100" (5kpc).

These observations reveal a galactic fountain in the hydrostatic limit. That is, the cooling time of the gas is longer than a sound-crossing time, so it is able to approach hydrostatic equilibrium before cooling. The pressure in the hot gas is $\mathrm{P} / \mathrm{k} \sim 14,000 \mathrm{~K} \mathrm{~cm}^{-3}$, which is similar to the value expected in the disk, so there appears to be pressure balance between the halo and disk, which would be expected if the flow time for hot material out of the disk occurred on a time shorter than the cooling time (the porosity of the disk is high enough to permit hot gas to flow into the halo easily). The cooling rate of the hot gas is approximately $0.1 \mathrm{M}_{\odot} \mathrm{yr}^{-1}$, which would recycle the disk gas in about a Hubble time.

It is gratifying that ROSAT has been able to, for the first time, detect galactic fountains in disk galaxies. However, it brings to light an important problem - why are these halos so faint (low luminosity)? The energy input from the ensemble of supernovae in NGC 891 (similar arguments can be made for NGC 4631) is $1 \times 10^{42} \mathrm{erg} \mathrm{s}^{-1}$, and most of the energy is expected to go into thermal energy. However, the observed X-ray luminosity from halo gas is < $1 \%$ of the energy released by supernovae. We can rule out the possibility that $10^{6}-10^{7} \mathrm{~K}$ gas is trapped in the disk since it would produce face-on spirals with very luminous diffuse emission, but face-on disks are faint as well (Fabbiano 1989). The other possibility is that hot gas is indeed produced, but it radiates most of its thermal energy at a temperature $<10^{6} \mathrm{~K}$, where ROSAT would have difficulty detecting such emission. 


\subsection{Starburst Galaxies}

A leading issue in the study of starburst galaxies is how the production of hot gas evolves in time, as this is expected to have profound consequences on star formation in the core and on the evolution of the superwind. Regarding star formation, the question can be raised as to whether the production of hot gas compresses the existing cold material, leading to an enormous enhancement of star formation. That is, does the hot gas initiate the starburst event, or it the hot gas merely a byproduct, having little influence on the star formation scenario? The second issue addresses the breakout of the hot material and its interaction with its environment. Some models suggest that, after breakout, the hot material flows along a column aligned with the minor axis, while other models suggest that the flow will be spherically symmetric (Chevalier and Clegg 1985; Tomisaka and Ikeuchi 1988). Since thorough discussions of a variety of starburst galaxies were presented in this conference by Heckman (1994) and Dahlem (1994), this contribution will describe results and interpretations of two other famous starburst galaxies, M82 and IC 342.

The early evolution of the hot gas in a starburst is expected to be spherical in nature (while still confined to the disk) and near pressure equilibrium with its surrounding. The thickness of the disk is typically $300 \mathrm{pc}$, so at a distance of the nearest starburst galaxies $(3-5 \mathrm{Mpc})$, the size of a young hot bubble would be only $10-20^{\prime \prime}$, which demands the use of the HRI if morphology is to be studied.

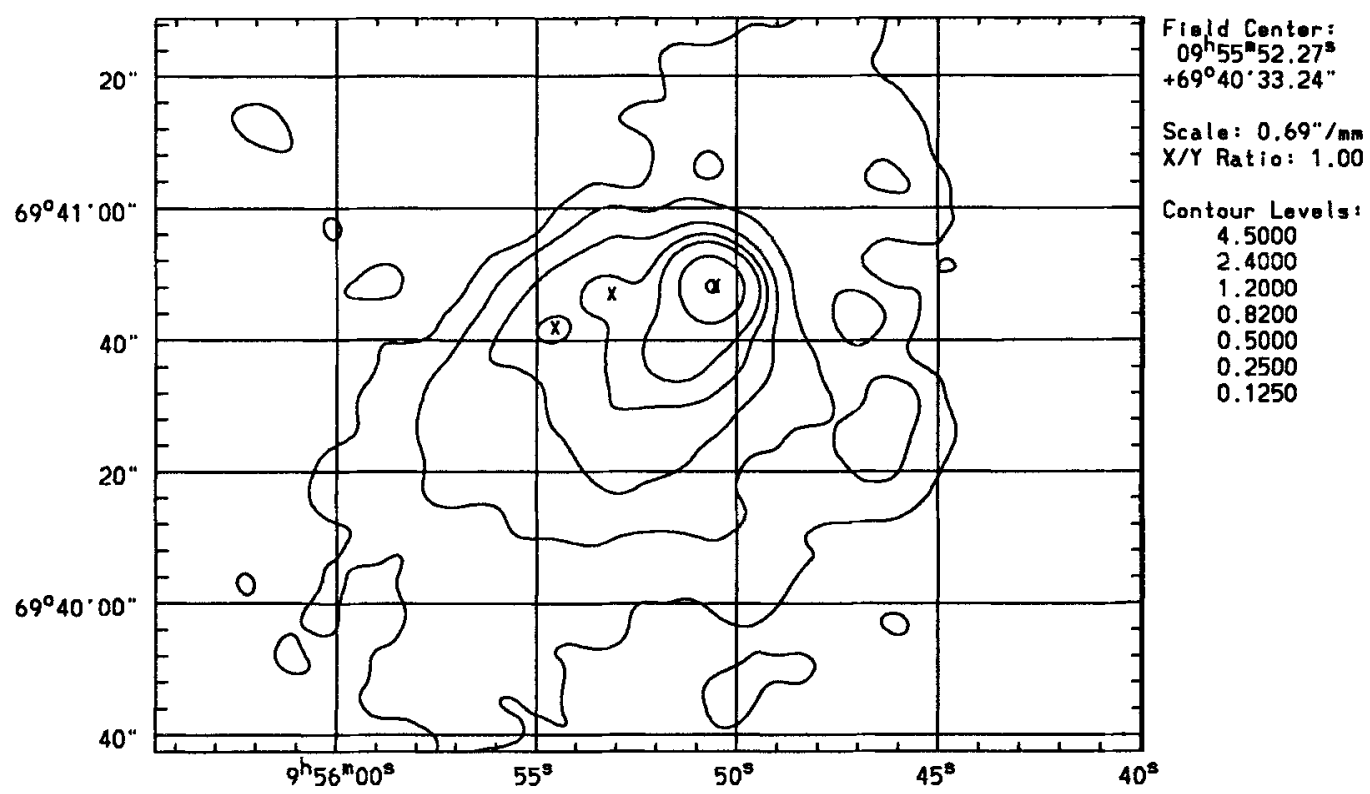

Figure 2a. The ROSAT HRI contour map of the center of M82 shows three point sources (marked by $\mathrm{X}$ ) plus diffuse emission extending along the minor axis of the galaxy, which is in the $\mathrm{N} / \mathrm{NW}$ direction. 


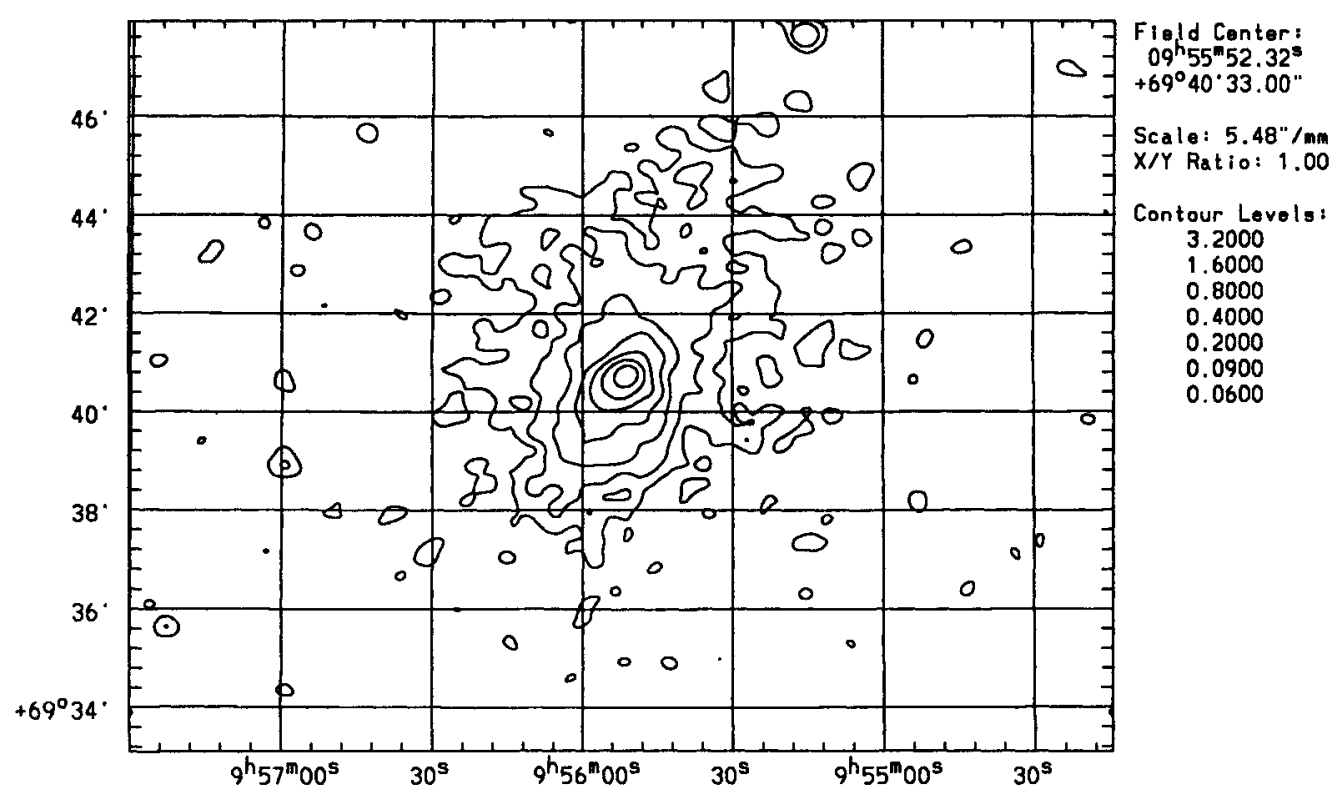

Figure 2b. The ROSAT HRI map of M82, smoothed to $20^{\prime \prime}$ resolution. The emission is seen to extend to at least $6^{\prime}$ to the north and to nearly $5^{\prime}$ to the south.

Our HRI observation of the starburst galaxy IC 342 reveals X-ray emission from the nuclear region that is fit with a point source plus extended diffuse emission of radius $200 \mathrm{pc}$ (Bregman, Cox, and Tomisaka 1993). The total luminosity from this central region is $1.5 \times 10^{39} \mathrm{erg} \mathrm{s}^{-1}$, of which half is due to the diffuse emission. For a filling factor of unity, the density in this diffuse gas is $0.2 \mathrm{~cm}^{-3}$, and the pressure is $\mathrm{P} / \mathrm{k} \sim 10^{6} \mathrm{~K} \mathrm{~cm}^{-3}$, which places this material close to the pressure of the molecular gas (Turner and Hurt 1992).

Based upon the bubble size, luminosity, and density, its age was inferred from theory and found to be quite young, approximately $2 \times 10^{5} \mathrm{yr}$. It is still confined to the disk and considerably smaller in extent than the intense molecular emission, which forms a bar nearly an arcminute in length $(1.3 \mathrm{kpc})$. This may suggest that star formation occurs over a region large compared to the $\mathrm{X}$-ray emission, in which case the $\mathrm{X}$-ray emission does not drive the starburst event, although it may enhance the star formation rate locally.

In M82, we find a more evolved starburst galaxy in which the hot gas bubble has broken out of the disk and is flowing away from the galaxy. Our HRI observation (Fig. 2a,b; Bregman, Schulman, and Tomisaka 1994), and the PSPC observation (Watson, private communication) reveal a bright core of X-ray emission, which extends in both directions along the minor axis to a maximum distance of about $7^{\prime}(7 \mathrm{kpc})$ from the nucleus. The HRI observation of the central region (Fig. 2a) shows three point sources present plus intense diffuse emission (point sources in M82 and their variability are discussed in 
Collura et al. 1994). For the inner $1^{\prime}(1 \mathrm{kpc})$, the $\mathrm{X}$-ray and $\mathrm{H} \alpha$ emission are closely correlated, and for a temperature near $1 \mathrm{keV}$, the hot gas pressure is $10^{7} .5 \mathrm{~K} \mathrm{~cm}^{-3}$, which is comparable to that deduced from the optical emission lines (Heckman, Armus, and Miley 1990), suggesting near pressure equilibrium between the two phases.

The extended emission is largely confined to cones that are nearly along the minor axis, although these are not cones of constant opening angle (Fig. 2b). The half-width at half-maximum of the cone, measured in degrees, decreases from $55-60^{\circ}$ in the inner $0.5^{\prime}$ to $20-30^{\circ}$ at a distance of about $1.5^{\prime}$ from the center. At distances beyond about $2^{\prime}$, the emission is consistent with having a constant opening angle. This change in the shape of the jet is consistent with it being confined by some surrounding medium close to the galaxy, but freely expanding further out.

Jet confinement and free expansion make different predictions for the change of X-ray surface brightness along the jet. Neglecting bandpass effects, the projected X-ray surface brightness, $S_{x}(r)$, in a freely expanding jet will depend upon the distance along the jet as $S_{x}(r) \propto r^{-3}$. In contrast, for a confined jet, the surface brightness is related to the width of the jet (d) by $S_{x} \propto d^{-3}$, so $S_{x}$ can decrease more slowly than $\mathrm{r}^{-3}$. For the case of M82, the surface brightness in the southern side decreases as expected for an adiabatically expanding flow in a partially confined jet. This may be true in the northern jet as well, although the analysis is complicated from absorption effects by the M82 disk and the morphology of the X-ray jet. Finally, adiabatically expanding gas should lead to a temperature drop, and increasingly softer emission is found outward along the jet (Watson, private communication; also seen in other starburst galaxies; see Heckman 1994). These observations support the outflow picture predicted from hydrodynamical considerations and indicate that a combination of confinement and free expansion offers the best explanation for the phenomenon.

JNB would like to acknowledge support for these research activities by NASA grants NAGW-2135 and NAG5-1955.

\section{References}

Bregman, J.N. 1980, ApJ, 236, 577.

Bregman, J.N., Cox, C.V., and Tomisaka, K. 1993, ApJ, 415, L79.

Bregman, J.N., and Glassgold, A.E. 1982, ApJ, 263, 564.

Bregman, J.N., and Pildis, R.A. 1992, ApJ, 398, L107.

Bregman, J.N., and Pildis, R.A. 1994, ApJ, in press.

Bregman, J.N., Schulman, E., and Tomisaka, K. 1994, ApJ, submitted.

Brinks, E., and Bajaja, E. 1986, A\&A, 169, 14

Brinks, E., and Shane, W.W. 1984, A\&A Sup, 55, 179.

Cash, W., Charles, P., Bowyer, S., Walter, F., Garmire, G., and

Riegler, G. 1980, ApJ, 238, L71.

Chevalier, R.A., and Clegg, A.W. 1985, Nature, 317, 44.

Chevalier, R.A., and Oegerle, W.R. 1979, ApJ, 227, 398.

Collura, A., Reale, F., Schulman, E., and Bregman, J. 1994, ApJ, in press.

Cox, D.P., and Reynolds, R.J. 1987, ARAA, 25, 303.

Dahlem, M. 1994, in "The 1st Annual ROSAT Science Symposium and Data Analysis Workshop", held Nov. 8-10, 1993 at the Univ. of Maryland, in press.

Deul, E.R., and van der Hulst, J.M. 1986, A\&A Sup, 67, 509. 
Fabbiano, G. 1989, ARAA, 27, 87.

Fried, P.M., Nousek, J.A., Sanders, W.T., and Kraushaar, W.L. 1980, ApJ, 242, 987.

Heckman. T.M 1994, in "The 1st Annual ROSAT Science Symposium and Data Analysis Workshop", held Nov. 8-10, 1993 at the Univ. of Maryland, in press.

Heckman, T., Armus, L., and Miley, G.K. 1990, ApJSS, 74, 833.

Heiles, C. 1979, ApJ, 229, 533.

Heiles, C. 1984, ApJ Suppl., 55, 585.

Long, K.S., Gordon, S.M., Blair, W.P., and Charles, P.A. 1994, in "The 1st Annual ROSAT Science Symposium and Data Analysis Workshop", held Nov. 8-10, 1993 at the Univ. of Maryland, in press.

MacLow, M.-M., McCray, R., and Norman, M.L. 1989, ApJ, 337, 141.

McCray, R., and Kafatos, M. 1987, ApJ, 317, 190.

Murphy, R., and Chu, Y.-H. 1994, in "The 1st Annual ROSAT Science Symposium and Data Analysis Workshop", held Nov. 8-10, 1993 at the Univ. of Maryland, in press.

Pietsch, W. 1993, in the "The ISM In Galactic Halos: Current Views", held at STScI, August 10-12, 1993.

Plucinsky, P.P., Snowden, S.L., Aschenbach, B., Egger, R., Edgar, R., and McCammon, D. 1994, in "The 1st Annual ROSAT Science Symposium and Data Analysis Workshop", held Nov. 8-10, 1993 at the Univ. of Maryland, in press.

Rand, R.J., Kulkarni, S.R., and Hester, J.J. 1990, ApJ, 352, L1.

Rupen, M.P. 1991, AJ, 102, 48.

Sancisi, R., and Allen, R.J. 1979, A\&A, 74, 73.

Schlegel, E.M. 1994, in "The 1st Annual ROSAT Science Symposium and Data Analysis Workshop", held Nov. 8-10, 1993 at the Univ. of Maryland, in press.

Schulman, E., and Bregman, J.N. 1994, in "The 1st Annual ROSAT Science Symposium and Data Analysis Workshop", held Nov. 8-10, 1993 at the Univ. of Maryland, in press.

Singh, K.P., Nousek, J.A., Burrows, D.N., and Garmire, G.P. 1987, ApJ, 313, 185.

Snowden, S.L. 1994, in "The 1st Annual ROSAT Science Symposium and Data Analysis Workshop", held Nov. 8-10, 1993 at the Univ. of Maryland, in press.

Tenorio-Tagle, G., and Bodenheimer, P. 1988, ARAA, 26, 145.

Tomisaka, K., and Ikeuchi, S. 1986, PASJ, 38, 697.

Tomisaka, K. and Ikeuchi, S. 1988, ApJ, 330, 695.

Turner, J.L., and Hurt, R.L. 1992, ApJ, 384, 72.

Wang, Q.D. 1994, in "The 1st Annual ROSAT Science Symposium and Data Analysis Workshop", held Nov. 8-10, 1993 at the Univ. of Maryland, in press.

Wang, Q., and Helfand, D.J. 1991a, ApJ, 373, 497.

Wang, Q., and Helfand, D.J. 1991b, ApJ, 379, 327.

Watson, M.G., Stanger, V., and Griffiths, R.E. 1984, ApJ, 286, 144. 